\title{
Boosting genetic vaccination
}

DOI:

10.1038/nrd2184
DNA vaccines are, in principle, cheap, safe and highly specific, and their design is aided by the increasing knowledge of pathogen genomes and powerful predictive computer programs for identifying 'good vaccine candidates'. However, the development of new-generation DNA vaccines is currently hampered by their weak immunogenicity. Reporting in PNAS, Andreakos et al. now present an elegant strategy to enhance the effectiveness of genetic vaccination, in an approach that holds particular promise for diseases such as malaria, HIV and TB (each in desperate need of a vaccine), as well as cancer vaccination.

In their report, the authors took advantage of insights into the molecular machinery controlling antigen presentation by dendritic cells (DCs), which forms the limiting step in the generation of adaptive and long-lasting immunity. DCs not only present antigen fragments of the pathogen on their surface, but also upregulate co-stimulatory molecules and secrete a range of cytokines and chemokines to orchestrate the immune response. Andreakos et al. combined one of the molecular switches responsible for the induction of these inflammatory mediators, nuclear factor- $\mathrm{KB}$ (NF$\kappa B$ )-inducing kinase (NIK), with an antigen fragment (for experimental purposes, green fluorescent protein (GFP)), in a recombinant attenuated adenoviral vector.

In vitro experiments with murine and human DCs demonstrated that this strategy led to the activation of
NF- $\kappa B$ in NIK-GFP adenovirustargeted cells, by both the canonical and the non-canonical pathways. This resulted in upregulation of major histocompatibility complex (MHC) antigen presentation and induction of the co-stimulatory molecules CD80 and CD86, as well as the secretion of pro-inflammatory cytokines and chemokines, which are prerequisites for the induction of a strong, cellmediated immune response. DCs primed with NIK-GFP were also able to potently activate human GFP-specific cytotoxic T lymphocytes (CTL). In vivo, the NIK-augmented adenovector injected into the tailbase of mice enhanced the immune response against the vector-encoded antigen and shifted the immune response towards a $\mathrm{T}_{\mathrm{H}} 1$-type immune response. This was evident by increased immunoglobulin G2a levels, T-cell proliferation, interferon- $\gamma$ production and CTL responses, which were more potently induced by the NIK-GFP adenovector than by GFP protein combined with complete Freud's adjuvant, an efficacious $\mathrm{T}_{\mathrm{H}} 1$ inducing adjuvant.

This strategy offers great potential for genetic vaccine development, as NIK augments the natural pathway of antigen presentation in a highly localized and specific manner. The authors further propose that their approach might also enhance the efficacy of recently described adenovirus-based vaccines against Ebola virus and $\mathrm{H} 5 \mathrm{~N} 1 \mathrm{avian}$ flu. There is increased interest in these vaccines not only

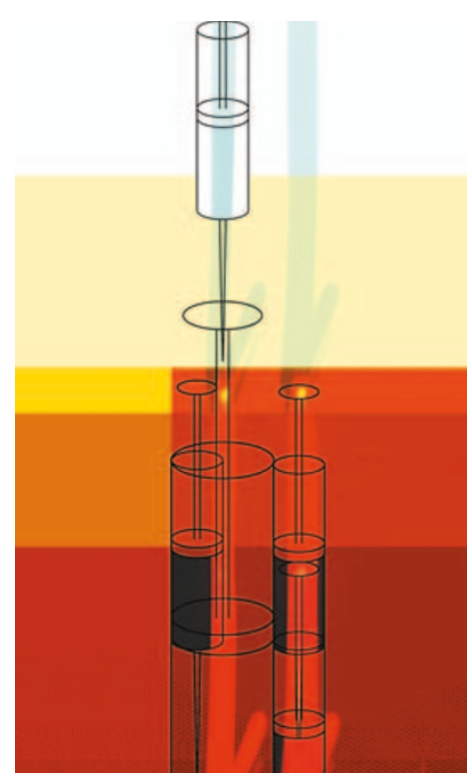

because of the deadly nature of the pathogens, but also the speed of the response induced by the adenoviral vectors and their ease of production. In addition, owing to the $\mathrm{T}_{\mathrm{H}} 1 / \mathrm{CTL}$ response induced, this approach might also be useful for cancer vaccines.

Alexandra Flemming

ORIGINAL RESEARCH PAPER Andreakos, E. et al. Activation of NF- $\mathrm{KB}$ by the intracellular expression of $\mathrm{NF}-\mathrm{KB}$ inducing kinase acts as a powerful vaccine adjuvant. Proc. Natl Acad Sci. USA 103, 14459-14464 (2006) FURTHER READING Sullivan, N. J. et al. Accelerated vaccination for Ebola virus haemorrhagic fever in non-human primates. Nature 424, 681-684 (2003) | Hoelscher, M. A. et al. Development of adenoviral-vector-based pandemic influenza vaccine against antigenically distinct human $\mathrm{H} 5 \mathrm{~N} 1$ strains in mice. Lancet 367 , 475-481 (2006) 